\title{
Adherence to sugars guidelines and micronutrient intakes in UK adult participants of the National Diet \& Nutrition Survey (2008/09-2011/12)
}

\author{
V.J. Burley, H.T. Lai and C.E.L. Evans \\ School of Food Science \& Nutrition, University of Leeds, Leeds, UK
}

The scientific literature on the relationship between added, free or non-milk extrinsic sugars and dietary micronutrient adequacy is not completely consistent but provides some evidence of micronutrient dilution at very high intakes ${ }^{(1)}$. However, of equal importance is the relationship at the lower extremes of intake, particularly in light of recent revised guidelines from the Department of Health in the UK that recommend limiting free sugars intake to no more than $5 \%$ of total dietary energy (TE) intake ${ }^{(2)}$. Analysis of the National Diet and Nutrition Survey Rolling Programme for 2008-2012 (NDNS RP) was undertaken to determine the relationship between intakes of non-milk extrinsic sugars (NMES), adherence to sugars guidelines and intakes of micronutrients, including iron, calcium, magnesium, potassium, zinc, iodine, vitamins A, C, B12 and D, riboflavin, and folate in adult participants.

Food diary data collected from 1692 adults (age 19-65 years) was analysed cross-sectionally using the NDNS RP dataset. The odds of consuming less than the lower reference nutrient intake were estimated by logistic regression analysis. Across the 5 categories of NMES intake, micronutrient intakes tended to be lowest in the extreme categories, that is, those consuming either $<5 \% \mathrm{TE}$ or greater than $20 \%$ TE from NMES. Compared with the lowest NMES consumers $(<5 \% \mathrm{TE})$, participants consuming 5-10\% TE from NMES had about half the odds of consuming less than the LRNI for riboflavin, $\mathrm{Mg}, \mathrm{K}$ and iodine, but those consuming more than $20 \%$ TE experienced 1.5 to 3 -fold greater odds for iron and zinc.
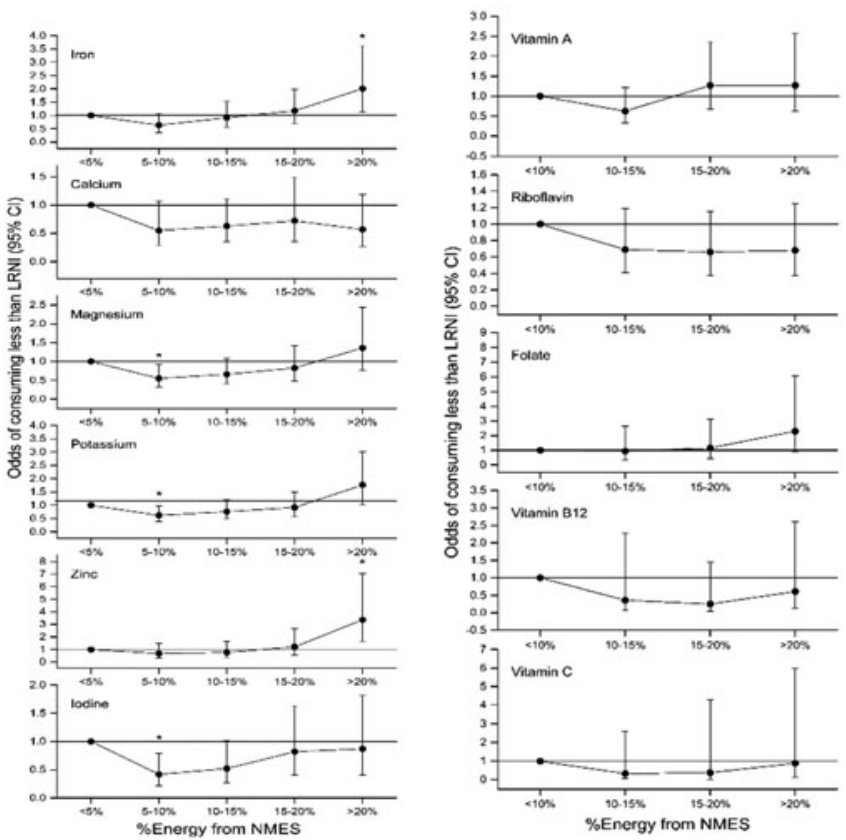

Energy adjusted odds of being a low micronutrient consumer associated with high NMES consumption were further elevated compared with the unadjusted analyses. However, the appropriateness of energy adjustment may be questioned given the view that a high intake of NMES may in itself elevate energy intakes. These findings confirm previous assertions that high intakes of NMES may be associated with micronutrient dilution but also that particular care should be taken to ensure an adequate consumption of micronutrients is achieved in those adhering to the revised guidelines on free sugars, notwithstanding the caution that should be applied in interpreting dietary data that is subject to a great deal of potential under reporting.

This work was supported by Sugar Nutrition UK. 